\title{
Targeted Drug Delivery with Focused Ultrasound-Induced Blood- Brain Barrier Opening Using Acoustically-Activated Nanodroplets
}

\author{
Cherry C. Chen ${ }^{a}$, Paul S. Sheeran ${ }^{b}$, Shih-Ying Wua ${ }^{a}$, Oluyemi O. Olumolade ${ }^{a}$, Paul A. \\ Dayton $^{\mathrm{b}}$, and Elisa E. Konofagou ${ }^{\mathrm{a}, \mathrm{c}}$ \\ aDepartment of Biomedical Engineering, Columbia University, New York, NY 10027 \\ bJoint Department of Biomedical Engineering, University of North Carolina and North Carolina \\ State University, Chapel Hill, NC, 27599 \\ 'Department of Radiology, Columbia University, New York, NY 10032
}

\section{Abstract}

Focused ultrasound (FUS) in the presence of systemically administered microbubbles has been shown to locally, transiently and reversibly increase the permeability of the blood-brain barrier (BBB), thus allowing targeted delivery of therapeutic agents in the brain for the treatment of central nervous system diseases. Currently, microbubbles are the only agents that have been used to facilitate the FUS-induced BBB opening. However, they are constrained within the intravascular space due to their micron-size diameters, limiting the delivery effect at or near the microvessels. In the present study, acoustically-activated nanodroplets were used as a new class of contrast agents to mediate FUS-induced BBB opening in order to study the feasibility of utilizing these nanoscale phase-shift particles for targeted drug delivery in the brain. Significant dextran delivery was achieved in the mouse hippocampus using nanodroplets at clinically relevant pressures. Passive cavitation detection was used in the attempt to establish a correlation between the amount of dextran delivered in the brain and the acoustic emission recorded during sonication. Conventional microbubbles with the same lipid shell composition and perfluorobutane core as the nanodroplets were also used to compare the efficiency of FUS-induced dextran delivery. It was found that nanodroplets had a higher BBB opening pressure threshold but a lower stable cavitation threshold than microbubbles, suggesting that contrast agent-dependent acoustic emission monitoring was needed. More homogeneous dextran delivery within the targeted hippocampus was achieved using nanodroplets without inducing inertial cavitation or compromising safety. Our results offered a new means of developing the FUS-induced BBB opening technology for potential extravascular targeted drug delivery in the brain, extending the potential drug delivery region beyond the cerebral vasculature.

\section{Introduction}

Focused ultrasound (FUS)-induced blood-brain barrier (BBB) opening has been shown to hold great promise for targeted drug delivery in the brain for the treatment of central nervous system diseases, including Alzheimer's [1], Huntington's [2] and Parkinson's [3] diseases

\footnotetext{
(C) 2013 Elsevier B.V. All rights reserved.
}

Publisher's Disclaimer: This is a PDF file of an unedited manuscript that has been accepted for publication. As a service to our customers we are providing this early version of the manuscript. The manuscript will undergo copyediting, typesetting, and review of the resulting proof before it is published in its final citable form. Please note that during the production process errors may be discovered which could affect the content, and all legal disclaimers that apply to the journal pertain. 
as well as brain cancers [4, 5]. Various therapeutic agents, including antibodies [6], neural stem cells [7], siRNA [2], chemotherapeutic molecules [8] and neurotrophic factors [3], have been delivered across the BBB and shown capable of inducing therapeutic effects using this technology. The FUS-microbubble-based drug delivery system relies on the capability of systemically-administered and acoustically-activated microbubbles to noninvasively, transiently and reversibly increase the cellular and vascular permeability of the BBB, thus allowing drug molecules that are normally restricted within the vasculature to cross the barrier and be delivered to the brain parenchyma. Previous studies by our group and others have shown that the extent of the BBB opening, as characterized by the opening volume, opening size and opening duration based on magnetic resonance or fluorescence imaging, is directly linked to the acoustic energy applied during sonication [9-12] and the physicochemical properties of the microbubbles used [6, 13, 14]. Informed selections of the acoustic exposure parameters and the type of contrast agent are critical for effective drug delivery while minimizing the possibility of side effects. Not accounting for these factors may result in unintended adverse bioeffects [15] or even undesired brain damage in more severe situations [9].

Real-time passive acoustic emissions from the oscillating microbubbles could offer means to identify signature characteristics that allow for the differentiation of the complex behaviors of microbubble cavitation during sonication [16-19]. During stable cavitation, the magnitude of microbubble oscillation depends on the acoustic pressure applied. Such behavior has been assumed to induce sufficient forces on the endothelium through either radiation or shear forces induced by microstreaming around the microbubbles [19]. Shear stress-induced endocytosis could also be a potential mechanism to increase the transcellular permeability [20]. As the acoustic energy is increased, larger radial microbubble expansion could induce inertial cavitation, leading to microbubble collapse and potentially generating liquid microjets to form pores within the cell membrane improving the cellular update of nanoparticles and drugs [21]. The disruption of the tight junction proteins between the adjacent brain endothelial cells during inertial cavitation could also increase the paracellular BBB permeability [22]. Regardless of the exact mechanism, the cavitation emission during sonication could be calculated and correlated with the outcome of the BBB opening, thus suggesting that the acoustic signature of the microbubbles could serve as a predictive indicator for estimating the amount of drug delivered to the targeted region [16, 23].

Currently, FUS-induced BBB opening has only been achieved using commercially available $[1,14,24]$ or in-house manufactured $[25,26]$ gaseous microbubbles. Despite their utility as mediating vehicles, microbubbles can only be used as vascular contrast agents since their size (typically $1-10 \mu \mathrm{m}$ ) prevents their extravasation, limiting the cavitation effects to regions at or near the vessels. Using fluorescence microscopy, our group [27] previously showed that homogeneous distribution of relatively larger dextran molecules (10 kDa and 70 $\mathrm{kDa}$ ) was more difficult to achieve than with smaller ones ( $3 \mathrm{kDa})$, highlighting the need for an agent with greater ability to induce molecular diffusion beyond the cerebral vasculature. In addition, gaseous microbubbles have short in vivo circulation half-life, typically on the order of minutes, thus requiring re-administration if repeated sonication is desired.

Phase-shift perfluorocarbon droplets may potentially offer the solution for such a demand $[28,29]$. Several groups have demonstrated the therapeutic utility of this alternative class of ultrasound contrast agents for applications such as targeted vessel occlusion [30], ablation enhancement [31], and drug delivery to solid tumors [32, 33]. Initially in the liquid state, nanoscale droplets show high stability in circulation (i.e., on the order of hours) and can be generated in sizes small enough to extravasate through leaky vasculature; but once exposed to sufficient rarefactional pressures they expand to form microbubbles capable of oscillation in a similar fashion as traditional microbubbles - ideal for ultrasound-mediated imaging and 
therapy. For the purposes of BBB opening, these agents may present a unique opportunity. Because droplet activation is dependent on the local rarefactional pressure, the microbubbles will only be generated within the narrow focal region where acoustic properties are the strongest, ensuring the therapeutic effect is limited to the desired treatment areas [34, 35]. Once initial BBB permeabilization is achieved, the droplets remaining will be small enough for potential extravasation during subsequent passes through the vasculature. Once entered into the interstitial space behind the barrier, the droplets could then be acoustically activated to form vaporized gaseous bubbles outside the constraints of the cerebral microvessels.

Therefore, the possibility exists for an extravascularly activated contrast agent for enhanced drug delivery at sites that are located deeply in the brain tissue or at regions with relatively low vasculature density. Activation of typical nanodroplet formulations requires acoustic pressures much higher than those used for FUS-induced BBB opening [36, 37], but recent studies have shown that it is possible to generate nanodroplets from highly volatile perfluorocarbons by pressurizing preformed microbubbles and condensing the gas core into liquid phase during slow cooling [23, 38]. This methodology can produce uniform nanodroplet size distributions with peak diameters near 200-300 nm that vaporize at acoustic pressures on the order of those required for BBB opening with microbubbles.

The main objective of the present study was to show the feasibility of utilizing phase-shift nanodroplets as the mediating agents for targeted drug delivery using FUS-induced BBB opening in vivo. Conventional in-house generated microbubbles with the same lipid shell composition and perfluorocarbon gaseous core were also used as the "standard" for comparing the efficiency of BBB opening based on targeted delivery of fluorescent dextran molecules. A series of clinically relevant acoustic exposure conditions were used to further demonstrate the safety of this technology for drug delivery in the brain.

\section{Materials and methods}

\subsection{Contrast agent generation}

Nanodroplets and microbubbles were formulated using the same lipid composition containing $90 \mathrm{~mol} \%$ 1,2-distearoyl-sn-glycero-3-phosphocholine (DSPC) and $10 \mathrm{~mol} \%$ 1,2distearoyl-sn-glycero-3-phosphoethanolamine-N-[methoxy(polyethylene glycol)2000] (DSPE-PEG2000) (Avanti Polar Lipids, Alabaster, AL). The perfluorobutane gas (PFB, 99 wt $\%$ purity) used for contrast agent generation was obtained from FluoroMed (Round Rock, TX). All chemicals were used as purchased without further purification.

Microbubbles were generated via mechanical agitation using a Vialmix shaker (BristolMyers-Squibb, New York, NY). A Multisizer III particle counter (Beckman Coulter, Opa Locka, FL) with a $30 \mu \mathrm{m}$ aperture was used to measure the microbubble suspension size distribution and concentration. Nanodroplets were generated via microbubble condensation, in which pre-formed microbubbles of volatile compounds were reverted to the liquid state by application of reduced temperature and increased ambient pressure [23, 38]. Briefly, PFB microbubbles were generated as described above and allowed to cool to room temperature. The vial containing the microbubbles was then immersed in a $\mathrm{CO}_{2}$ /isopropanol bath maintained at a temperature between $-7{ }^{\circ} \mathrm{C}$ and $-10{ }^{\circ} \mathrm{C}$ for approximately $1 \mathrm{~min}$. The vial was subsequently connected to an adjustable air-pressure supply, and the headspace pressure inside the microbubble vial increased $30-70 \mathrm{kPa}$ for approximately $30 \mathrm{~s}$ to facilitate condensation. A Malvern Nano Zetasizer (Malvern Instruments Ltd., Malvern, Worcestershire, U.K.) was used to measure the size distribution of the droplet emulsion generated. 


\subsection{In vitro acoustic nanodroplet vaporization setup}

Acoustic nanodroplet vaporization was investigated in vitro using an experimental setup described previously [39]. An ultra-high-speed framing camera with a 24-frame buffer (SIMD24; Specialised Imaging, Simi Valley, CA) was interfaced with an inverted microscope (IX71; Olympus, Center Valley, PA) with a 100× (NA=1.0) water immersion objective. An acryliclined, continuously degassed water bath was mounted to the microscope and maintained at $37^{\circ} \mathrm{C}$. The optical resolution of the system allowed observation of particles larger than approximately $500 \mathrm{~nm}$. A $1 \mathrm{MHz}$ spherically-focused piston transducer with a $2.2 \mathrm{~cm}$ diameter and a focal length of $3.75 \mathrm{~cm}$ (IL0106HP; Valpey Fisher Corp., Hopkinton, MA) was aligned with the optical focus by use of a calibrated needle hydrophone (HNA-0400; Onda Corp., Sunnyvale, CA). The transducer was driven by sinusoidal 20-cycle pulses generated by a manuallytriggered arbitrary waveform generator (AFG 3101; Tektronix, Inc., Beaverton, OR) amplified 60 dB (A500; ENI, Rochester, NY). The sinusoid amplitude was adjusted to change the peak rarefactional pressure experienced by the droplets in focus. The manual trigger was synchronized with the input of the highspeed camera in order to simultaneously capture video of droplet vaporization. Droplet emulsions were diluted 50\% in phosphate-buffered saline (PBS) and pumped through a nearly optically and acoustically transparent microcellulose tube (Spectrum Laboratories, Inc., Greensboro, NC). The focal plane of the tube was controlled via a 3-axis micropositioner (MMO-203; Narishige Group, East Meadow, NY). The ultra-high-speed camera was set to operate at 20 million frames per second with a $30 \mathrm{~ns}$ exposure such that many frames could be captured within a single cycle of the ultrasound pulse. The transducer was calibrated at focus by the needle hydrophone (HNA-0400).

\subsection{Animal preparation}

All experiments were performed in accordance with procedures approved by the Columbia University Institutional Animal Care and Use Committee. A total of 80 male C57BL/6 mice (Harlan Laboratories; Indianapolis, IN) weighing 20-25 g were used for this study. The animals were divided into two experimental groups using either nanodroplets (group \#1) or microbubbles (group \#2) as the contrast agents for FUS-induced BBB opening. The groups were further divided into 14 cohorts based on the experimental protocol as listed in Table 1. Before sonication, each mouse was anesthetized using 1-2\% isoflurane-oxygen mixture (SurgiVet, Smiths Medical PM; Norwell, MA) and its hair on the scalp was removed with an electric clipper and a depilatory cream. A modified 27 -gauge $1 \frac{1}{2}$ butterfly catheter (Terumo Medical; Somerset, NJ) was inserted into the tail vein for contrast agent injection. The animal body temperature was maintained throughout the experiment using a heated pad.

\subsection{In vivo BBB opening ultrasound setup}

The experimental setup, as shown in Fig. 1, was used as previously described [26, 27]. A single-element, spherical FUS transducer (center frequency: $1.5 \mathrm{MHz}$; focal depth: $60 \mathrm{~mm}$; Imasonic, Besancon, France) was driven by a function generator (Agilent Technologies, Palo Alto, CA) through a $50 \mathrm{~dB}$ power amplifier (E\&I, Rochester, NY). A confocal pulseecho transducer (center frequency: $10 \mathrm{MHz}$; focal length: $60 \mathrm{~mm}$; Olympus NDT, Waltham, MA), which was attached to a computer-controlled 3D positioning system (Velmex, Lachine, QC, Canada), was used to target specific brain structures following procedures published previously [14]. The pulse-echo transducer, which was driven by a pulser-receiver system with a $20 \mathrm{~dB}$ amplification in the receive-only mode (Model 5800; Olympus NDT), was connected to a digitizer (Gage Applied Technologies, Lachine, QC, Canada) to passively acquire acoustic emissions from activated nanodroplets and microbubbles within $\pm 1 \mathrm{~V}$ input range. 
The peak-rarefactional pressure profile used in the present study was estimated based on previously performed calibrations done in degassed water with a $0.2 \mathrm{~mm}$ diameter needle hydrophone (Precision Acoustics; Dorchester, Dorset, UK) [14]. The pressure amplitude values were corrected to account for $18.1 \%$ attenuation through the murine skull [14], whereas the axial and lateral full-widths at half-maximum intensities of the beam were 7.5 $\mathrm{mm}$ and $1 \mathrm{~mm}$, respectively. The acoustic exposure reported throughout the present study is given in terms of the peak-rarefactional pressure amplitudes.

\subsection{In vivo BBB opening protocol}

Pulsed FUS (pulse length: $6.7 \mathrm{~ms}$; pulse repetition frequency: $5 \mathrm{~Hz}$; duration: $5 \mathrm{~min}$ ) at acoustic pressures ranging between 0.15 and $0.60 \mathrm{MPa}$ was applied transcranially to the targeted left hippocampus of the mouse brain while the right hippocampus served as the control. Prior to any contrast agent administration, a 30-s sonication using the same acoustic parameters was applied in order to measure the baseline background signal needed in the acoustic emission analysis, as described below. The injected contrast agent samples were freshly diluted before each animal injection. For group \#1, a $60 \mu \mathrm{L}$ nanodroplet suspension was first diluted at $50 \mathrm{vol} \%$ using PBS to an estimated final concentration of $5 \times 10^{9} \mathrm{\#} / \mathrm{mL}$ based on $100 \%$ condensation during production. It was then co-administered, via bolus injection, with $60 \mu \mathrm{L} 3 \mathrm{kDa}$ dextran solution (Life Technologies; Carlsbad, CA) at $2 \mathrm{mg} / \mathrm{mL}$ concentration through the tail vein $10 \mathrm{~s}$ prior to each sonication. For group \#2, the microbubble suspension was diluted in PBS to a final concentration of $8 \times 10^{8} \# / \mathrm{mL}$, and a 60 $\mu \mathrm{L}$ of the diluted microbubble sample, together with $60 \mu \mathrm{L}$ dextran solution was bolus injected following the same protocol. In addition, two sham cohorts to which no ultrasound was applied were also injected with either nanodroplets or microbubbles and fluorescent dextrans to serve as the basis for comparison in the fluorescence imaging analysis (see description below).

A 1-h period was allowed after sonication to enable the dextran to circulate throughout the vasculature and diffuse into the brain parenchyma. At the end of the allotted time, the animal was sacrificed by transcardiac perfusion using $30 \mathrm{~mL}$ PBS for $5 \mathrm{~min}$ followed by $60 \mathrm{~mL} 4 \%$ paraformaldehyde for $8 \mathrm{~min}$. The mouse brain was extracted from the skull, post-fixed in $4 \%$ paraformaldehyde overnight, and then prepared for either frozen $(60 \mu \mathrm{m})$ or paraffin $(6$ $\mu \mathrm{m})$ sections. The frozen sections were used to analyze fluorescence intensity in order to determine the BBB opening magnitude, while the paraffin sections were used to study the safety of the procedure through histological examinations of representative brain samples from each treatment cohort via hematoxylin and eosin (H\&E) staining. The histology image interpretation was performed blindly, i.e., without knowledge of the FUS exposure parameters.

\subsection{Fluorescence imaging analysis}

Bright-field and epi-fluorescence images of the brain sections were captured using an Olympus DP30BW digital camera mounted on an upright Olympus BX61 microscope. The extent of BBB opening was determined based on the quantification of dextran delivery into the targeted hippocampus. The fluorescence intensity measurement was similarly performed following our previously published protocol [40]. Briefly, a section representing the ventraldorsal midline, as determined by anatomical landmarks, was first selected, and 4 adjacent sections were then selected on either the dorsal or the ventral side of the midline. The sonicated (left) and the control (right) hippocampus was manually outlined using MATLAB (The Mathworks; Natick MA), and the spatial average of fluorescence intensity in the region of interest (ROI) was calculated using ImageJ (National Institutes of Health, Bethesda, MD). The relative fluorescence enhancement was calculated by dividing the difference in fluorescence intensity between the left and right ROIs by the spatial average of 
the right hippocampus. A fluorescence intensity threshold, defined as twice that of the standard deviation of the control ROI, was applied on each image in order to separate the fluorescence signal from dextran molecules from the background tissue autofluorescence. For each brain, the reported fluorescence enhancement was thus approximated as the sum of the relative fluorescence intensity from all 9 sections. Successful dextran delivery for an individual brain was concluded if the fluorescence enhancement was higher by two standard deviations relative to the average of the corresponding sham cohort. The fluorescence enhancement value for each of the 12 experimental conditions was obtained by averaging all mice sonicated under the same acoustic exposure. $100 \%$ opening efficiency was determined when all the mice evaluated in each cohort showed significant dextran delivery.

\subsection{Acoustic emission analysis}

The acoustic emission analysis was performed similarly to that described previously [26]. In order to quantify the acoustic responses of the vaporized nanodroplets or microbubbles, two cavitation parameters were calculated: stable cavitation dose (SCD) and inertial cavitation dose (ICD). The SCD, which was associated with the stable nonlinear oscillation of the contrast agents, was quantified based on the peak amplitude of the spectra around each harmonic frequency of each pulse in the range between 3 and $9 \mathrm{MHz}$ over the first minute sonication duration. The ICD, which was associated with the inertial energy of the collapsing microbubbles, was quantified based on the broadband emission after filtering the harmonic and ultraharmonic signals using a comb filter with rectangular rejection bands centered around the harmonic and ultraharmonic frequencies (rejection bandwidths of 350 $\mathrm{kHz}$ and $100 \mathrm{kHz}$, respectively) across the same frequency range over the same sonication duration. The net emission from the contrast agents could then be determined by subtracting the background signal measured using the same acoustic exposure prior to nanodroplet or microbubble administration.

\subsection{Statistical analysis}

An unpaired two-tailed Student's t-test was performed to evaluate the significance of the fluorescence enhancement between the sonicated and the control hippocampus under each of the acoustic exposures. In addition, unpaired two-tailed Student's t-tests were used to determine the significance of the acoustic emission responses from the contrast agents across different sonication pressures. All statistical analysis was performed using GraphPad Prism (La Jolla, CA).

\section{Results}

\subsection{Contrast agent generation}

The PFB phase-shift nanodroplet emulsions appeared partially translucent by visual inspection and were similar in stability and size to previously reported results [38]. Fig. 2A shows a representative number-weighted size distribution obtained by averaging 3 separate samples ( 3 measurements per sample). The size distributions measured in the present in vivo study were similar across all samples, and fell within the instrumental uncertainty of the Zetasizer. The averaged number-weighted mean, median and mode diameters across all samples were $204 \pm 10 \mathrm{~nm}, 209 \pm 29 \mathrm{~nm}$ and $180 \pm 25 \mathrm{~nm}$, respectively.

The mechanical agitation method produced opaque milky microbubble suspensions that were stable during the experimental timeframe. Fig. 2B shows a representative numberweighted size distribution of the microbubble sample as measured by Multisizer III. The size distributions among all microbubble suspensions used throughout the study were found to be statistically the same, and the averaged number-weighted mean, median and mode diameters were $1.36 \pm 0.32 \mu \mathrm{m}, 1.08 \pm 0.23 \mu \mathrm{m}$ and $0.86 \pm 0.35 \mu \mathrm{m}$, respectively. 


\subsection{In vitro acoustic nanodroplet vaporization}

In order to confirm the nanodroplets generated via microbubble condensation were acoustically vaporizable, high-speed optical microscopy was used to visually verify the vaporization of individual stationary droplets at pressures relevant to our in vivo experiment. Fig. 3 shows that at peak-rarefactional pressure of approximately $0.35 \mathrm{MPa}$, no detectable number of vaporized bubbles appeared within the focal plane. However, when the pressure amplitude was increased to $0.45 \mathrm{MPa}$, vaporized bubbles were observed almost immediately upon exposure to the ultrasound beam. These acoustically activated bubbles were within the micrometer size range and appeared to be acoustically responsive (i.e., they could expand and contract according to the pressure change during a single pulse). The activation of submicron droplets was also repeatedly observed at higher pressures (up to 1.1 MPa tested in the current study) using the in vitro setup, complementing our finding of the in vivo pressure threshold of BBB opening using nanodroplets (see discussion below).

\subsection{In vivo FUS-induced BBB opening}

Using fluorescently-labeled $3 \mathrm{kDa}$ dextran as a model drug molecule, the extent of the FUSinduced BBB opening could be quantified as the relative fluorescence enhancement in the sonicated hippocampus over the control. The two sham cohorts, for which no ultrasound was applied, did not show any change in fluorescence intensity between the two hemispheres (Fig. S1). Quantified fluorescence enhancement results confirmed this observation as no detectable increase in fluorescence intensity between the two ROIs was calculated (Table 2). For the rest of the 12 experimental conditions, the measured fluorescence enhancement was compared to their corresponding sham cohort in order to determine whether sufficient amount of dextran molecules were delivered into the targeted region.

Following the systemic administration of nanodroplets and subsequent BBB opening, a significant increase in dextran delivery in the targeted ROI was observed at 0.45 and 0.60 MPa (60\% opening efficiency with $\mathrm{P}=0.047$ and $100 \%$ opening efficiency with $\mathrm{P}=0.0002$, respectively) while only up to $33 \%$ of the animals evaluated showed a significant fluorescence enhancement when sonicated at pressures below 0.45 MPa (Table 2). Due to the inconsistency across animals, there was no statistically significant $(\mathrm{P}>0.56)$ increase in fluorescence enhancement to clearly indicate BBB opening with dextran delivery for sonication pressures ranging between 0.15 and $0.30 \mathrm{MPa}$ (Fig. 4A). At pressures above 0.45 $\mathrm{MPa}$, fluorescence was observed not only within or near large vessels, but also diffusely distributed across the hippocampi (Figs. 5D and 5E).

Following the systemic administration of microbubbles and subsequent BBB opening, a significant fluorescence enhancement was detected for all mice when sonicated at pressures including and above $0.30 \mathrm{MPa}(\mathrm{P}<0.0050)$. A similar inconsistency of dextran delivery across animals was seen at $0.225 \mathrm{MPa}$ that only 3 out of 5 mice showed significant fluorescence enhancement in the targeted ROI (Table 2). Thus, no sufficient statistical difference $(\mathrm{P}>0.16)$ could be obtained to unequivocally show BBB opening at pressures below $0.30 \mathrm{MPa}$ (Fig. 4B). The detectable fluorescence signal was predominately contained within vessels at $0.30 \mathrm{MPa}$ but more diffusely distributed throughout the targeted region at $0.45 \mathrm{MPa}$, indicating a more homogenous distribution of dextran molecules (Figs. 5H and $5 \mathrm{I})$. At $0.60 \mathrm{MPa}$, heterogeneous spots of particularly high levels of fluorescence in combination with diffusely distributed fluorescence was observed (Fig. 5J).

For each acoustic pressure, microbubbles produced greater fluorescence enhancement compared to nanodroplets. The normalized fluorescence enhancement with pressure amplitude followed a linear relationship with correlation coefficients at 0.76 and 0.94 for nanodroplets and microbubbles, respectively. The pressure threshold, at which significant 
fluorescence enhancement was detected in comparison to the sham animals, was higher for the nanodroplets $(0.60 \mathrm{MPa})$ than that for the microbubbles $(0.30 \mathrm{MPa})$, although the percent enhancement values at the threshold pressures were not statistically different $(\mathrm{P}=0.56)$.

\subsection{Contrast agent-dependent BBB opening threshold}

Fig. 6 shows the quantitative acoustic emission results detected before and after the contrast agent administrations at various sonication pressures. For nanodroplets, the quantified SCD showed significant increase $(\mathrm{P}<0.0001)$ at $0.60 \mathrm{MPa}$ (Fig. 6A), corresponding to the significant dextran delivery detected based on fluorescence microscopy. However, no statistical difference was determined at $0.45 \mathrm{MPa}(\mathrm{P}=0.21)$ despite a $90 \%$ mean signal increase after nanodroplet administration owing to the large variations among different mice. The SCD increase followed a linear relationship with the sonication pressure $\left(\mathrm{R}^{2}=0.99\right)$. The quantified ICD, on the other hand, showed no detectable inertial cavitation dose across all pressures (Fig. 6B), implying that no significant vaporized nanodroplet fragmentation was detected during sonication.

The acoustic emission results for the microbubble group are shown in Figs. 6C and 6D. Interestingly, the SCD showed significant increase $(\mathrm{P}<0.0005)$ for all pressure levels after microbubble injections regardless the outcome of the BBB opening (Fig. 6C). Similar to the nanodroplet group, the SCD increase followed a linear correlation with the FUS pressure amplitude $\left(R^{2}=0.93\right)$. The ICD measurement showed significant signal increase after microbubble injection at $0.60 \mathrm{MPa}(\mathrm{P}=0.017)$ but not at other pressure levels, indicating microbubbles underwent inertial cavitation during sonication only at the highest acoustic energy exposure (Fig. 6D).

In order to test whether acoustic emissions could be used to predict the magnitude of the BBB opening, the relative fluorescence enhancement was plotted against the SCD for all sonications using each contrast agent (Fig. 7). Both agents showed relatively good linear correlations between these two parameters $\left(\mathrm{R}^{2}=0.74\right.$ for nanodroplets and $\mathrm{R}^{2}=0.67$ for microbubbles). A cavitation dose opening threshold can also be derived when SCD was grouped based on whether significant dextran delivery was detected based on fluorescence enhancement [16] (Fig. 8). For the mice sonicated in the presence of nanodroplets, a statistically higher $(\mathrm{P}<0.0001)$ stable cavitation emission was detected for cases where significant fluorescence enhancement was measured. The highest SCD value, at which no evidence of dextran delivery was detected, was $74 \mathrm{~V} \cdot \mathrm{s}$. Out of the 10 animals that showed significant fluorescence increase, 3 mice (30\%) had their SCD lower than $74 \mathrm{~V} \cdot \mathrm{s}$, suggesting that this level could be used as the threshold for predicting successful FUS-induced BBB opening using nanodroplets as the contrast agents.

Interestingly, the acoustic threshold of BBB opening appeared to be contrast agent dependent. For the microbubble group, the highest SCD value, for which no detectable dextran delivery was observed, was $1.4 \mathrm{kV} \cdot \mathrm{s}$, significantly higher $(\mathrm{P}<0.0001)$ than the SCD threshold found for the nanodroplet group. A much more prominent threshold was held for group \#2, for 1 out of 15 mice (6.7\%) with significant fluorescence enhancement had a SCD lower than $1.4 \mathrm{kV} \cdot \mathrm{s}$. Despite the wider range of the calculated SCDs, in which successful BBB opening was detected, a statistical difference was obtained $(\mathrm{P}=0.04)$ between cases with or without significant dextran delivery. The threshold for predicting FUS-induced BBB opening in the presence of microbubbles was therefore concluded to be $1.4 \mathrm{kV} \cdot \mathrm{s}$ (Fig. 8). 


\subsection{Safety}

Histological evaluation was performed in order to assess for potential tissue damage caused by the procedure. Fig. 9 shows the bright-field microscopic images taken of representative brain samples at 0.45 and $0.60 \mathrm{MPa}$. These two pressure amplitudes were chosen since relatively consistent BBB opening was detected only at these pressure levels for both nanodroplet and microbubble groups. Close examinations did not reveal any discrete damage sites, such as clusters of dark neurons, small erythrocyte extravasations, hemorrhage or microvacuolations at $0.45 \mathrm{MPa}$ for either the nanodroplet or the microbubble group (Fig. 9 top). A few $(<10)$ dark neurons were identified from 3 nonadjacent sections of the representative brain sample that was sonicated at $0.30 \mathrm{MPa}$ using nanodroplets (Fig. S2). No other tissue damage indicators were observed, indicating that this could be an artifact due to inadequate perfusionfixation [41]. However, small clusters of extravasated erythrocytes in addition to a few dark neurons were observed for a sample from the microbubble/0.60 MPa cohort (Fig. 9O). Less than 10 clusters were found throughout the sonicated region across all sections, indicating minor tissue damage. The larger portion of the targeted hippocampus appeared to be normal and the neurons appeared to be unaffected (Fig. 9M).

\section{Discussion}

The current study utilized acoustically-activated nanodroplets as a new class of contrast agents to facilitate targeted drug delivery in the brain after FUS-induced BBB opening in mice. Though similar agents have been used in other therapeutic applications, to the best of our knowledge, this is the first study that has explored the benefits of a nanodroplet-based approach to FUS-induced BBB opening. Using fluorescent dextran as a model drug, the extent of the BBB opening was quantified and compared between nanodroplets and the conventional contrast agent, i.e., microbubbles (Figs. 4 and 5). The two agents were compositionally the same - having lipid-encapsulated perfluorobutane cores - but the nanodroplet approach afforded taking advantage of the benefits of both the liquid and gaseous state of the cores. The acoustic emission generated from the contrast agents during sonication was recorded and analyzed in order to gain insights to their cavitation characteristics (Fig. 6). A linear correlation between BBB permeabilization, as indicated by the relative fluorescence enhancement within the targeted hippocampus, and the acoustic emission, as characterized by the SCD, was found for both nanodroplets $\left(R^{2}=0.74\right)$ and microbubbles $\left(\mathrm{R}^{2}=0.67\right)$ (Fig. 7). Interestingly, the acoustic threshold, at which significant dextran delivery was observed, appeared to be contrast agent dependent (Fig. 8). Our results suggested future contrast agent-specific monitoring during FUS-induced BBB opening might be needed.

The high-speed microscopy results confirmed that the nanodroplet samples were acoustically vaporizable at exposure conditions similar to that used during BBB opening in vivo (Fig. 3). The vaporization pressure threshold detected in vitro corresponded to our in vivo estimation remarkably well. While it was possible that nanodroplet vaporization at pressures lower than $0.45 \mathrm{MPa}$ could still occur, it was more likely that the number of activated nanodroplets was too low to cause any detectable fluorescence enhancement. Thus, nanodropletmediated dextran delivery to the targeted hippocampus could not be consistently achieved until the sonication pressure was higher than $0.45 \mathrm{MPa}$ (Table 2). For the purpose of direct comparison, the droplets used in this study were formed from perfluorobutane. While the pressures used were sufficient to cause some droplet vaporization, other studies have shown that highly efficient vaporization at frequencies near $1 \mathrm{MHz}$ required pressures on the order of $1 \mathrm{MPa}$ or greater [23, 42]. Further studies are needed to develop different nanodroplet formulations in order to decrease the in vivo vaporization threshold by incorporating more volatile perfluorocarbons, such as perfluoropropane [38]. Additionally, 
the not-yet-optimized droplet formulation could also explain the lower fluorescence enhancement observed using nanodroplets than that of microbubbles even though the injection amount of the former was much greater (Fig. 4).

Previous work reported by our group and others have been focusing on increasing the delivered dose and distribution by optimizing ultrasonic parameters and microbubble characteristics, including peak-rarefactional pressure [10], pulse repetition frequency [40], pulse length [27] and microbubble size [25, 26]. Choi et al. [27] showed that homogenous distribution of $3 \mathrm{kDa}$ dextran molecules was possible by incorporating short ultrasonic pulses to allow sufficient microbubble replenishment at the focus during sonication. The utilization of acoustically vaporized nanodroplets in this paper produced a similar homogenous dextran distribution throughout the targeted volume and was more prominent when compared to microbubbles under the same acoustic exposure settings (Figs. 5D-5J). Similar to our previous studies, fluorescence was not only detected within or near large vessels but also diffusely distributed within the hippocampus, thus showing greater promise for delivery of therapeutic agents to a wider range of cellular targets outside the microvasculature. As a preliminary feasibility study, the sonication parameters used in the current paper were simply adapted based on our previous FUS-induced BBB opening experiments using microbubbles. Therefore, it is possible that nanodroplet-specific pulse sequence could be custom designed to further enhance the delivery of larger drug molecules. Since short pulse lengths appeared to be adequate to activate nanodroplets at clinically relevant pressures [39] (Fig. 3), it is possible that higher delivery doses and a more homogenous distribution could be achieved using short pulses with nanodroplets as well [27]. In addition, it is possible that a higher but safer sonication pressure could be achieved with nanodroplets since the threshold for inducing inertial cavitation was higher than that of microbubbles (Fig. 6).

Initial histological examinations confirmed the safety for the nanodroplet-mediated FUSinduced BBB opening technology. The case where a few dark neurons were identified for the nanodroplet/0.30 MPa brain sample was believed to be an isolated incident that was more likely due to inadequate perfusion-fixation [41] (Fig. S2). In comparison to nanodroplets, microbubble-mediated BBB opening resulted minor tissue damage at the targeted region for both brain samples evaluated from the 0.60 MPa cohort (Fig. 9O). Based on the H\&E staining results, it is reasonable to conclude that the significantly increased dextran delivery at $0.60 \mathrm{MPa}$ was to be caused by the increased magnitude of disruption at the focal region. However, such molecular delivery enhancement with compromised safety may not be desirable in the clinic.

Passively detected acoustic emissions originated from the oscillating contrast agents could be used to characterize the type of cavitation events occurred during sonication, providing insights to the mechanism of the FUS-induced BBB opening. Only an increase in the harmonics and ultraharmonics signals detected after nanodroplet administration at all pressures, suggesting that the vaporized nanodroplet underwent mainly nonlinear stable cavitation during sonication. The quantified SCD showed significant increase at the highest pressure amplitude $(0.60 \mathrm{MPa})$, indicating that a minimum SCD threshold was needed to induce BBB opening. Interestingly, when microbubbles were used as the contrast agent, significant SCD increase was seen across all sonication pressures regardless of the outcome of BBB opening. The SCD value for the microbubble group at the lowest pressure (902 V·s at $0.15 \mathrm{MPa}$ ) was much higher than that for the nanodroplet group even at the highest pressure (353 V.s at $0.60 \mathrm{MPa}$ ), suggesting that the SCD threshold was contrast agentdependent. This result was further demonstrated in Fig. 8, in which significant difference was calculated between threshold baselines (i.e., negative cases for which no dextran delivery was observed). This finding was very important for acoustic emission based BBB 
opening monitoring since contrast agent-specific thresholds would then need to be investigated separately. The significant increase in ICD for microbubbles at $0.60 \mathrm{MPa}$ in combination with the minor tissue damage observed based on histological evaluation also suggested that inertial cavitation could be used as a safety indicator to avoid undesired bioeffects.

The linear correlation between the calculated SCD and the relative fluorescence enhancement was slightly weaker for microbubbles than that for nanodroplets (Fig. 7). Two factors could be contributing to the dissociation between these two parameters for microbubblemediated BBB opening. First, our passive cavitation detection was sensitive to signals arising from any microbubbles in the beam path of the transducers, including those that were outside the focal region. For those microbubbles, it was possible that their contribution to the nonlinear acoustic emission signal was not proportionate to their contribution to the fluorescence enhancement, and therefore weakening the correlation between these two parameters. On the other hand, the acoustic emission signal during nanodroplet-mediated BBB opening only came from those nanodroplets vaporized within the focal region, as opposed to the inherent signal from the off-targeted microbubbles. This could also explain the significantly lower BBB opening threshold determined based on acoustic emission for nanodroplets (Fig. 8). Secondly, in the present study, the extent of the BBB opening was determined based on intensity measured using fluorescence microscopy. Despite the fact that precautions were taken during image capturing to minimize overexposure, it might be possible that signal saturation could still occur after large amount of dextran delivery. As a result, the level of the BBB opening might be underestimated at higher pressures, especially for the microbubble group (Fig. 5J).

Based on optical microscopy, Sheeran et al. [23] previously reported that it was possible to have microbubbles formed from acoustically vaporized nanodroplets larger than those in the original parent microbubble suspension. One possible explanation for such an increase in size was bubble fusion or Ostwald ripening resulted from vaporization of nanodroplets in very close proximity at high concentration. The resulting increased size distribution of the activated nanodroplets could attribute to the lower SCD threshold for BBB opening using nanodroplets than that with microbubbles (Fig. 8) [26]. In addition, the larger bubbles formed from vaporized nanodroplets could amplify the shear stress induced during stable cavitation to other neighboring cells along the microvessels [13] at higher efficiency than traditional smaller microbubbles, thus increasing the number of BBB disrupted sites to facilitate a more homogenous dextran delivery distribution without the concern of causing undesired inertial cavitation at higher pressures (Figs. 5D and 5E). Finally, it is also possible that the vaporization of the volatile perfluorocarbon droplets used here introduced additional mechanical effects beyond the standard microbubble cavitation effects, aiding permeabilization without being registered as ICD or SCD. Recent studies have shown that the expansion of less volatile perfluorocarbon droplets occurs on a microsecond timescale [43], but little is known on the magnitude of the shear stresses introduced by the rapid expansion of more highly volatile droplets, such as those formed from perfluorobutane.

Our initial results utilizing nanodroplets as the mediating agent for FUS-induced BBB opening offer a new means of developing this technology for potential extravascular targeted drug delivery in the brain parenchyma. Our findings indicate that a potential advantage for employing nanodroplets as the contrast agent is that they would only be activated within the transducer focal zone when sufficient acoustic energy is reached. This yields the possibility of enhancing specificity through focusing of the ultrasound beam, thus promising more spatial and temporal control of the targeting procedure to minimize undesired thermal and cavitation-based bioeffects. Moreover, it eliminates the "noise" signal generated from non-activated nanodroplets, allowing more accurate monitoring of BBB 
opening based on passive acoustic emission detection (Fig. 7). Additionally, the nanodroplets could potentially be conjugated with targeting ligands to allow for more specific active-targeting applications on the molecular and cellular level [44]. The previously described post-labeling technique [45] for generating targeted microbubbles could easily be translated to produce targeted nanodroplets. This "shake and bake" methodology offers the possibility of developing the nanodroplet-mediated FUS-induced BBB opening technology for potentially a large number of molecular targets for future personalized nanomedicine.

\section{Conclusion}

In this paper, we have performed an initial feasibility study to explore the utilization of phase-shift nanodroplets for targeted drug delivery in the brain with FUS-induced BBB opening. Significant dextran delivery was achieved in the mouse hippocampus using acoustically-activated nanodroplets at clinically relevant pressure amplitudes. Passive cavitation detection was used in the attempt to establish a correlation between the amount of dextran delivered in the brain and the acoustic emission recorded during sonication. Conventional microbubbles with the same lipid shell composition and perfluorobutane core as the nanodroplets were also used to compare the efficiency of FUS-induced dextran delivery. It was found that nanodroplets had a higher BBB opening pressure threshold but a lower stable cavitation threshold than the microbubbles. More homogeneous dextran delivery within the targeted hippocampus was achieved using nanodroplets without inducing inertial cavitation or compromising safety. Our results offered a new means of developing the FUS-induced BBB opening technology for potential extravascular targeted drug delivery in the brain.

\section{Supplementary Material}

Refer to Web version on PubMed Central for supplementary material.

\section{Acknowledgments}

The authors would like to thank Dr. Yao-Sheng Tung at the University of Southern California for his insightful discussions, and Dr. Terry Matsunaga at the University of Arizona for collaborations in nanodroplet development that led to the formulations used in this study. We also appreciate the assistance of Dr. John Hossack and Ali Dhanaliwala at the University of Virginia in the use of the ultra-high speed imaging system. This research was supported in part by the National Institutes of Health (NIH) grants R01 EB009041 (E.E.K.), R01 AG038961 (E.E.K.), and R21 EB011704 to Dr. Terry Matsunaga with P.A.D., as well as pilot funds from National Science Foundation (NSF) DMR 1122483 (P.A.D.) and a NSF Graduate Research Fellowship to P.S.S. The ultra-high speed imaging system was purchased through NIH shared instrumentation grant S10 RR025594.

\section{References}

1. Choi JJ, Wang SG, Brown TR, Small SA, Duff KEK, Konofagou EE. Noninvasive and transient bloodbrain barrier opening in the hippocampus of Alzheimer's double transgenic mice using focused ultrasound. Ultrasonic Imaging. 2008; 30:189-200. [PubMed: 19149463]

2. Burgess A, Huang YX, Querbes W, Sah DW, Hynynen K. Focused ultrasound for targeted delivery of siRNA and efficient knockdown of Htt expression. J. Control. Release. 2012; 163:125-129. [PubMed: 22921802]

3. Baseri B, Choi JJ, Deffieux T, Samiotaki G, Tung YS, Olumolade O, Small SA, Morrison B, Konofagou EE. Activation of signaling pathways following localized delivery of systemically administered neurotrophic factors across the blood-brain barrier using focused ultrasound and microbubbles. Phys. Med. Biol. 2012; 57:N65-N81. [PubMed: 22407323] 
4. Park EJ, Zhang YZ, Vykhodtseva N, McDannold N. Ultrasound-mediated blood-brain/blood-tumor barrier disruption improves outcomes with trastuzumab in a breast cancer brain metastasis model. J. Control. Release. 2012; 163:277-284. [PubMed: 23000189]

5. Ting CY, Fan CH, Liu HL, Huang CY, Hsieh HY, Yen TC, Wei KC, Yeh CK. Concurrent bloodbrain barrier opening and local drug delivery using drug-carrying microbubbles and focused ultrasound for brain glioma treatment. Biomaterials. 2012; 33:704-712. [PubMed: 22019122]

6. Jordao JF, Ayala-Grosso CA, Markham K, Huang YX, Chopra R, McLaurin J, Hynynen K, Aubert I. Antibodies Targeted to the Brain with Image-Guided Focused Ultrasound Reduces Amyloid-beta Plaque Load in the TgCRND8 Mouse Model of Alzheimer's Disease. Plos One. 2010; 5

7. Burgess A, Ayala-Grosso CA, Ganguly M, Jordao JF, Aubert I, Hynynen K. Targeted delivery of neural stem cells to the brain using MRI-guided focused ultrasound to disrupt the blood-brain barrier. Plos One. 2011; 6

8. Park J, Zhang YZ, Vykhodtseva N, Jolesz FA, McDannold NJ. The kinetics of blood brain barrier permeability and targeted doxorubicin delivery into brain induced by focused ultrasound. J. Control. Release. 2012; 162:134-142. [PubMed: 22709590]

9. Samiotaki G, Vlachos F, Tung YS, Konofagou EE. A quantitative pressure and microbubble-size dependence study of focused ultrasound-induced blood-brain barrier opening reversibility in vivo using MRI. Magn. Reson. Med. 2012; 67:769-777. [PubMed: 21858862]

10. Chopra R, Vykhodtseva N, Hynynen K. Influence of exposure time and pressure amplitude on blood-brainbarrier opening using transcranial ultrasound exposures. Acs Chem Neurosci. 2010; 1:391-398. [PubMed: 20563295]

11. McDannold NJ, Vykhodtseva NI, Hynynen K. Microbubble contrast agent with focused ultrasound to create brain lesions at low power levels: MR imaging and histologic study in rabbits. Radiology. 2006; 241:95-106. [PubMed: 16990673]

12. Mesiwala AH, Farrell L, Wenzel HJ, Silbergeld DL, Crum LA, Winn HR, Mourad PD. Highintensity focused ultrasound selectively disrupts the blood-brain barrier in vivo. Ultrasound Med. Biol. 2002; 28:389-400. [PubMed: 11978420]

13. Park J, Fan ZZ, Deng CX. Effects of shear stress cultivation on cell membrane disruption and intracellular calcium concentration in sonoporation of endothelial cells. J Biomech. 2011; 44:164169. [PubMed: 20863503]

14. Choi JJ, Pernot M, Small SA, Konofagou EE. Noninvasive, transcranial and localized opening of the blood-brain barrier using focused ultrasound in mice. Ultrasound Med. Biol. 2007; 33:95-104. [PubMed: 17189051]

15. Choi JJ, Pernot M, Brown TR, Small SA, Konofagou EE. Spatio-temporal analysis of molecular delivery through the blood-brain barrier using focused ultrasound. Phys. Med. Biol. 2007; 52:5509-5530. [PubMed: 17804879]

16. Arvanitis CD, Livingstone MS, Vykhodtseva N, McDannold N. Controlled ultrasound-induced blood-brain barrier disruption using passive acoustic emissions monitoring. Plos One. 2012; 7

17. McDannold N, Vykhodtseva N, Hynynen K. Use of ultrasound pulses combined with definity for targeted blood-brain barrier disruption: A feasibility study. Ultrasound Med. Biol. 2007; 33:584590. [PubMed: 17337109]

18. Tung YS, Vlachos F, Choi JJ, Deffieux T, Selert K, Konofagou EE. In vivo transcranial cavitation threshold detection during ultrasound-induced blood-brain barrier opening in mice. Phys. Med. Biol. 2010; 55:6141-6155. [PubMed: 20876972]

19. McDannold N, Vykhodtseva N, Hynynen K. Targeted disruption of the blood-brain barrier with focused ultrasound: association with cavitation activity. Phys. Med. Biol. 2006; 51:793-807. [PubMed: 16467579]

20. Afadzi M, Strand S, Nilssen E, Masoy S-E, Johansen T, Hansen R, Angelsen B, de L Davies C. Mechanisms of the ultrasound-mediated intracellular delivery of liposomes dextrans, IEEE transactions on ultrasonics. ferroelectrics and frequency control. 2013; 60:21-33.

21. Bekeredjian R, Kroll RD, Fein E, Tinkov S, Coester C, Winter G, Katus HA, Kulaksiz H. Ultrasound targeted microbubble destruction increases capillary permeability in hepatomas. Ultrasound Med. Biol. 2007; 33:1592-1598. [PubMed: 17618040] 
22. Sheikov N, McDannold N, Vykhodtseva N, Jolesz F, Hynynen K. Cellular mechanisms of the blood-brain barrier opening induced by ultrasound in presence of microbubbles. Ultrasound Med. Biol. 2004; 30:979-989. [PubMed: 15313330]

23. Sheeran PS, Luois S, Dayton PA, Matsunaga TO. Formulation and acoustic studies of a new phase-shift agent for diagnostic and therapeutic ultrasound. Langmuir. 2011; 27:10412-10420. [PubMed: 21744860]

24. Hynynen K, McDannold N, Vykhodtseva N, Jolesz FA. Noninvasive MR imaging-guided focal opening of the blood-brain barrier in rabbits. Radiology. 2001; 220:640-646. [PubMed: 11526261]

25. Choi JJ, Feshitan JA, Baseri B, Wang SG, Tung YS, Borden MA, Konofagou EE. MicrobubbleSize Dependence of Focused Ultrasound-Induced Blood-Brain Barrier Opening in Mice In Vivo. IEEE Trans. Biomed. Eng. 2010; 57:145-154. [PubMed: 19846365]

26. Tung YS, Vlachos F, Feshitan JA, Borden MA, Konofagou EE. The mechanism of interaction between focused ultrasound and microbubbles in blood-brain barrier opening in mice. J. Acoust. Soc. Am. 2011; 130:3059-3067. [PubMed: 22087933]

27. Choi JJ, Selert K, Vlachos F, Wong A, Konofagou EE. Noninvasive and localized neuronal delivery using short ultrasonic pulses and microbubbles. P Natl Acad Sci USA. 2011; 108:1653916544.

28. Sheeran PS, Dayton PA. Phase-Change Contrast Agents for Imaging and Therapy. Curr Pharm Design. 2012; 18:2152-2165.

29. Zhao YZ, Lu CT, Li XK, Cai J. Ultrasound-mediated strategies in opening brain barriers for drug brain delivery. Expert opinion on drug delivery. 2013

30. Zhang M, Fabiilli ML, Haworth KJ, Fowlkes JB, Kripfgans OD, Roberts WW, Ives KA, Carson PL. Initial investigation of acoustic droplet vaporization for occlusion in canine kidney. Ultrasound Med. Biol. 2010; 36:1691-1703. [PubMed: 20800939]

31. Zhang M, Fabiilli ML, Haworth KJ, Padilla F, Swanson SD, Kripfgans OD, Carson PL, Fowlkes JB. Acoustic droplet vaporization for enhancement of thermal ablation by high intensity focused ultrasound. Acad. Radiol. 2011; 18:1123-1132. [PubMed: 21703883]

32. Rapoport N, Nam KH, Gupta R, Gao ZG, Mohan P, Payne A, Todd N, Liu X, Kim T, Shea J, Scaife C, Parker DL, Jeong EK, Kennedy AM. Ultrasound-mediated tumor imaging and nanotherapy using drug loaded block copolymer stabilized perfluorocarbon nanoemulsions. J. Control. Release. 2011; 153:4-15. [PubMed: 21277919]

33. Rapoport NY, Kennedy AM, Shea JE, Scaife CL, Nam KH. Controlled and targeted tumor chemotherapy by ultrasound-activated nanoemulsions/microbubbles. J. Control. Release. 2009; 138:268-276. [PubMed: 19477208]

34. Phillips LC, Puett C, Sheeran PS, Matsunaga TO, Dayton PA. Phase-shift perfluorocarbon agents enhance high intensity focused ultrasound (HIFU) thermal delivery with reduced near-field heating. J. Acoust. Soc. Am. In Press

35. Puett C, Phillips LC, Sheeran PS, Dayton PA. In vitro parameter optimization for spatial control of focused ultrasound ablation when using low boiling point phase-change nanoemulsions. Journal of Therapeutic Ultrasound. 2013

36. Williams R, Wright C, Cherin E, Reznik N, Lee M, Gorelikov I, Foster FS, Matsuura N, Burns PN. Characterization of submicron phase-change perfluorocarbon droplets for extravascular ultrasound imaging of cancer. Ultrasound Med Biol. 2013

37. Zhang P, Porter T. An in vitro study of a phase-shift nanoemulsion: a potential nucleation agent for bubble-enhanced HIFU tumor ablation. Ultrasound Med. Biol. 2010; 36:1856-1866. [PubMed: 20888685]

38. Sheeran PS, Luois SH, Mullin LB, Matsunaga TO, Dayton PA. Design of ultrasonicallyactivatable nanoparticles using low boiling point perfluorocarbons. Biomaterials. 2012; 33:32623269. [PubMed: 22289265]

39. Sheeran PS, Wong VP, Luois S, McFarland RJ, Ross WD, Feingold S, Matsunaga TO, Dayton PA. Decafluorobutane as a phase-change contrast agent for low-energy extravascular ultrasonic imaging. Ultrasound Med. Biol. 2011; 37:1518-1530. [PubMed: 21775049] 
40. Choi JJ, Selert K, Gao Z, Samiotaki G, Baseri B, Konofagou EE. Noninvasive and localized bloodbrain barrier disruption using focused ultrasound can be achieved at short pulse lengths and low pulse repetition frequencies. J Cereb Blood Flow Metab. 2011; 31:725-737. [PubMed: 20842160]

41. Jortner BS. The return of the dark neuron. A histological artifact complicating contemporary neurotoxicologic evaluation. Neurotoxicology. 2006; 27:628-634. [PubMed: 16650476]

42. Sheeran PS, Matsunaga TO, Dayton PA. Phase-transition thresholds and vaporization phenomena for ultrasound phase change nanoemulsions assessed via high speed optical microscopy. Phys. Med. Biol. 2013 In Review.

43. Wong ZZ, Kripfgans OD, Qamar A, Fowlkes JB, Bull JL. Bubble evolution in acoustic droplet vaporization at physiological temperature via ultra-high speed imaging. Soft Matter. 2011; 7:4009-4016.

44. Sheeran PS, Streeter JE, Mullin LB, Matsunaga TO, Dayton PA. Toward ultrasound molecular imaging with phase-change contrast agents: An in-vitro proof-of-principle. Ultrasound Med Biol. 2013 In Press.

45. Chen CC, Borden MA. Ligand conjugation to bimodal poly(ethylene glycol) brush layers on microbubbles. Langmuir. 2010; 26:13183-13194. [PubMed: 20695557] 


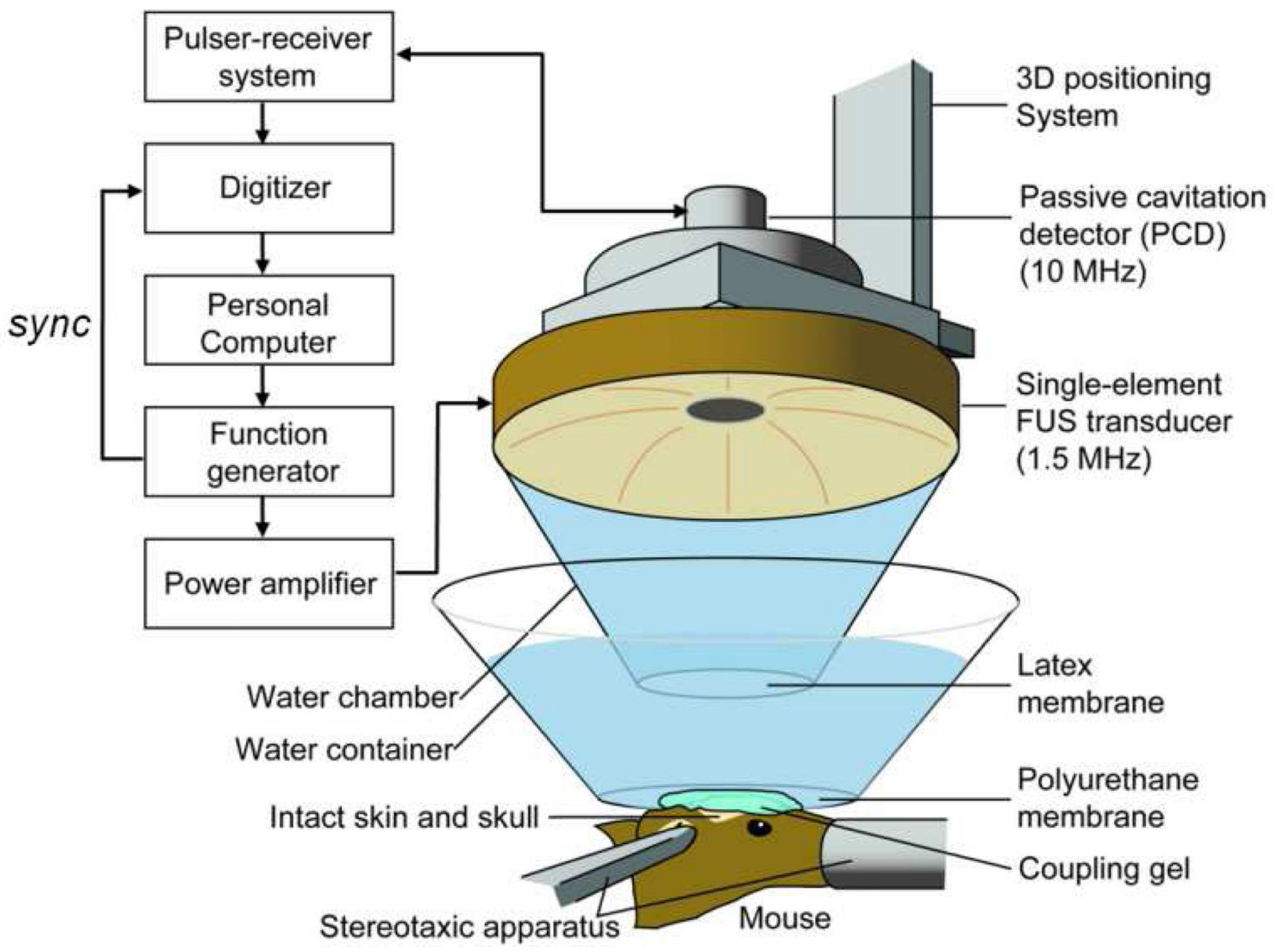

Fig. 1.

Experimental setup of in vivo FUS-induced BBB opening and transcranial cavitation detection. 

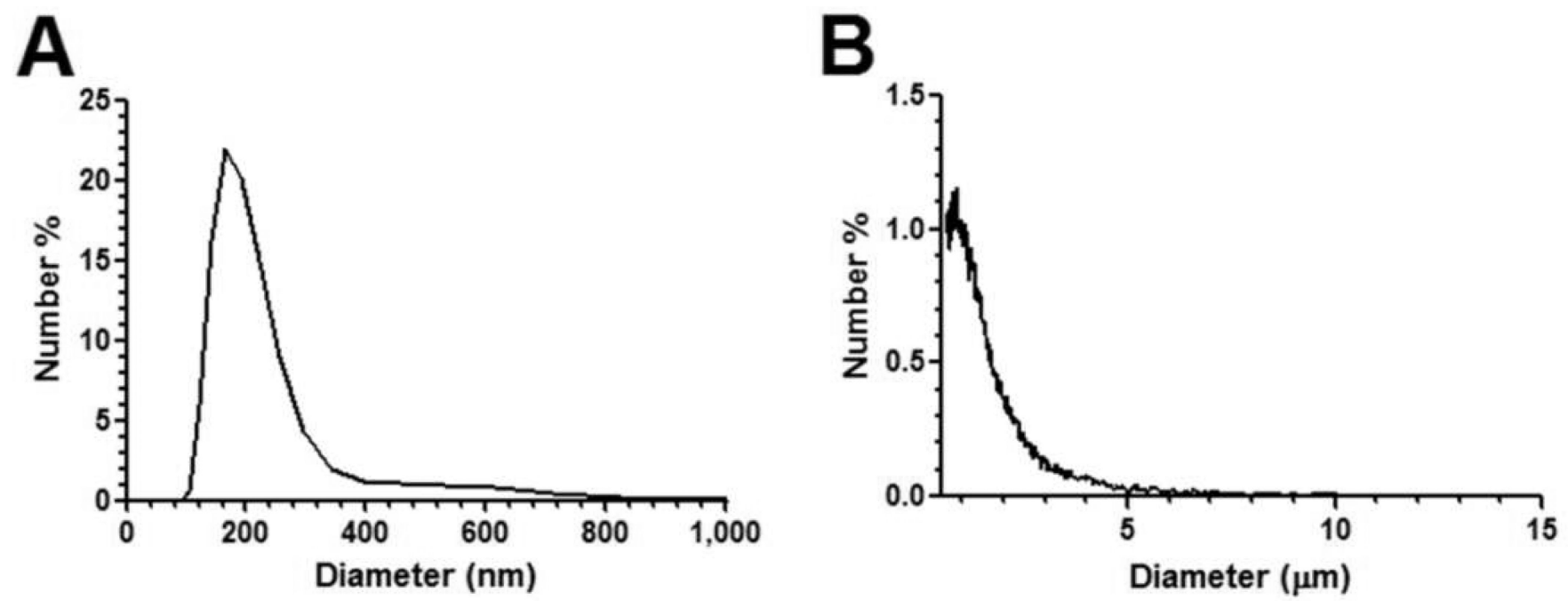

Fig. 2.

Representative size distributions of nanodroplet (A) and microbubble (B) suspensions as measured by Malvern Zetasizer and Coulter Multisizer, respectively. (A) The averaged number-weighted mean, median and mode diameters for the nanodroplet sample shown were $206 \pm 93 \mathrm{~nm}, 190 \mathrm{~nm}$ and $164 \mathrm{~nm}$, respectively. All nanodroplet samples were diluted by 50 vol\% prior to injection. (B) The averaged number-weighted mean, median and mode diameters for the microbubble sample shown were $1.36 \pm 0.02 \mu \mathrm{m}, 1.10 \mu \mathrm{m}$ and $0.79 \mu \mathrm{m}$, respectively. All microbubble samples were diluted to $8 \times 10^{8}$ particle/mL prior to injection. 


\section{$0.35 \mathrm{MPa}$}

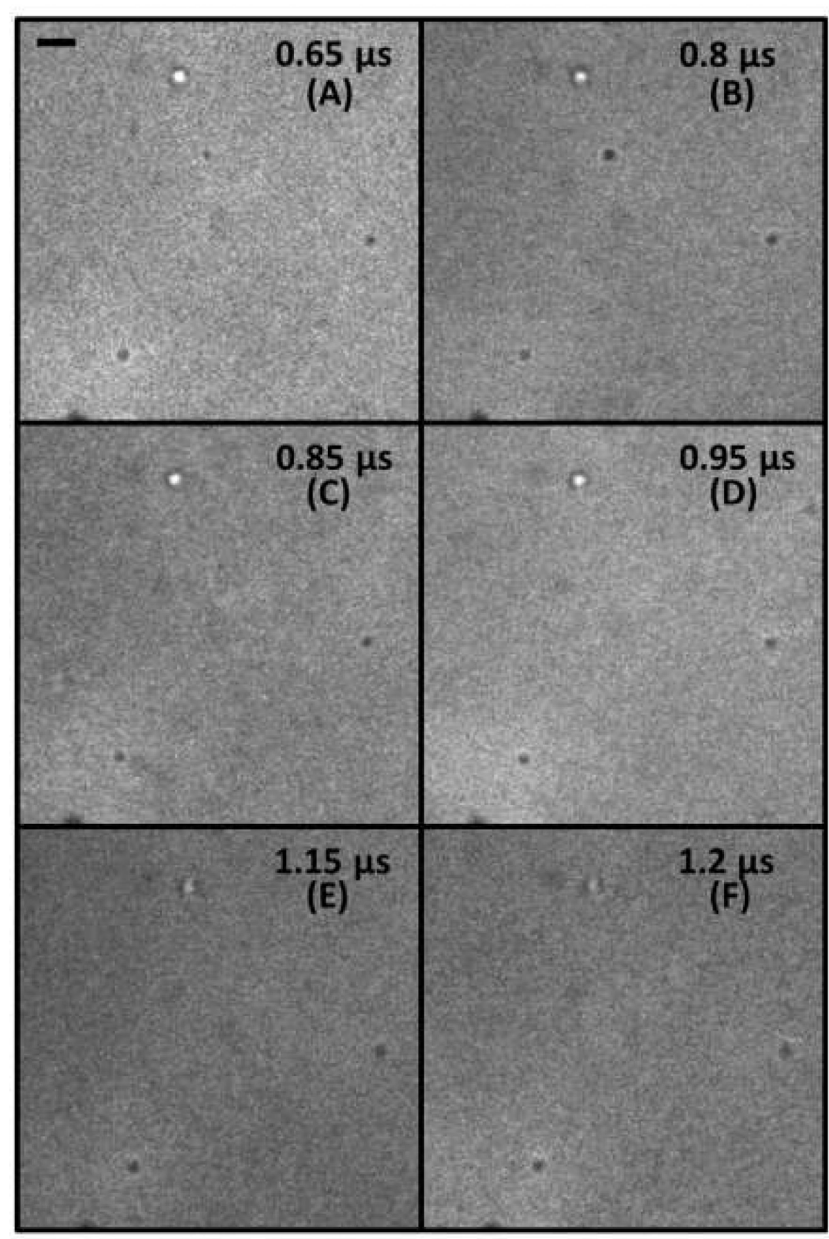

\section{$0.45 \mathrm{MPa}$}

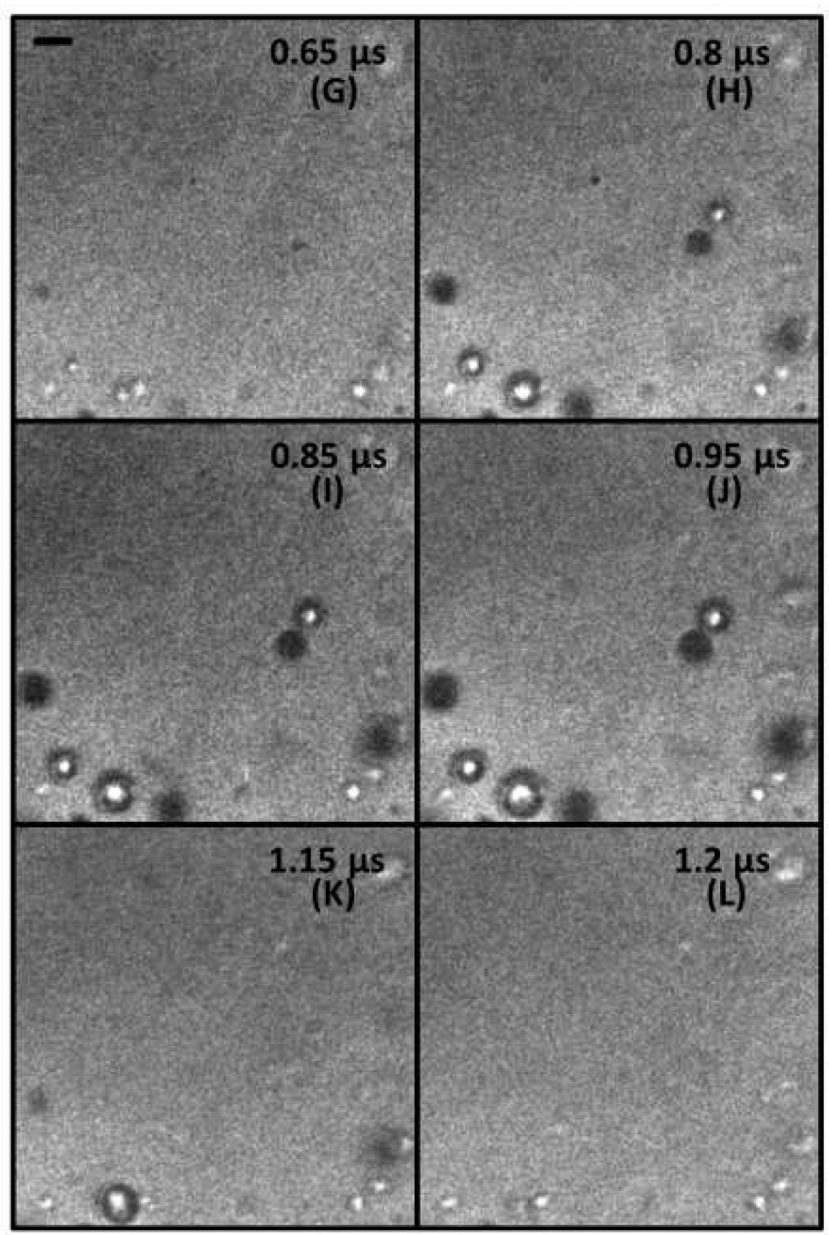

Fig. 3.

Still-frame images showing nanodroplet activation within the first two cycles of the pulse as a function of sonication pressure. Frames A-D and G-J were taken in the first rarefactional half-cycle, while $\mathrm{E}-\mathrm{F}$ and $\mathrm{K}-\mathrm{L}$ were taken in the following compressional cycle. No nanodroplet vaporization was detected at $0.35 \mathrm{MPa}$ (left) while micron-scale acousticallyresponsive bubbles were seen expanding in the rarefactional cycle at $0.45 \mathrm{MPa}(\mathrm{G}-\mathrm{J})$. When transitioned to the next positive pressure cycle $(\mathrm{K}, \mathrm{L})$, the newly formed bubbles were observed to respond to the acoustic pressure by compressing until no longer visible in the field of view. Times shown are relative to the start of the pulse, and the scale bars indicate 5 $\mu \mathrm{m}$. 
A

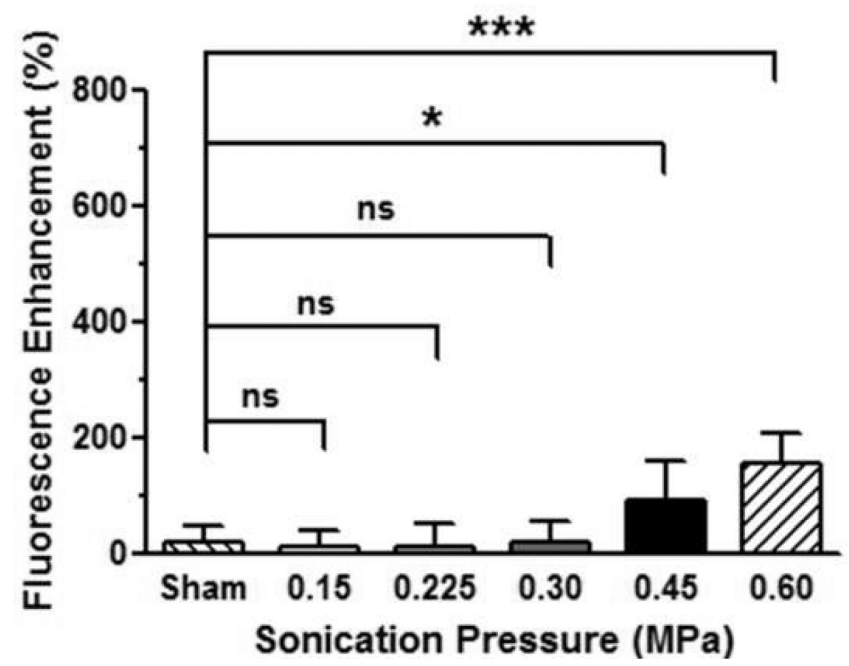

B

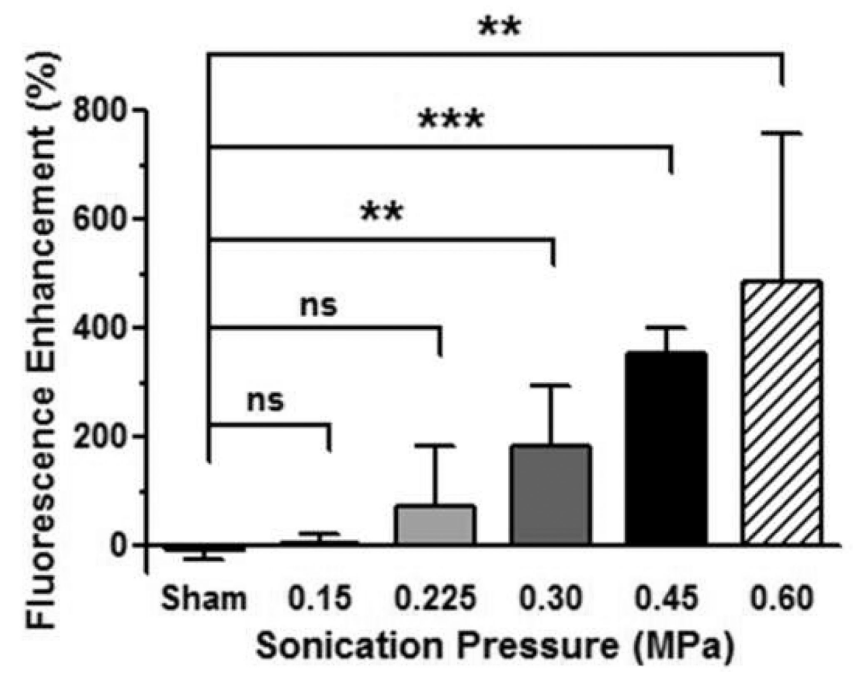

Fig. 4.

Normalized fluorescence enhancement between the targeted and the control hippocampi using either nanodroplets (A) or microbubbles (B) to mediate BBB opening at distinct sonication pressures. The normalized fluorescence enhancement with pressure followed a linear relationship with correlation coefficient $\mathrm{R}^{2}$ being 0.76 and 0.94 for nanodroplets and microbubbles, respectively. Significant dextran delivery was observed at pressures higher than 0.45 MPa using nanodroplets while the BBB opening threshold was decreased down to $0.30 \mathrm{MPa}$ when microbubbles were administered. All numbers are reported as mean \pm standard deviation. (ns: not significant; *: $\mathrm{P}<0.05$; **: $\mathrm{P}<0.01$; ***: $\mathrm{P}<0.001$ ) 


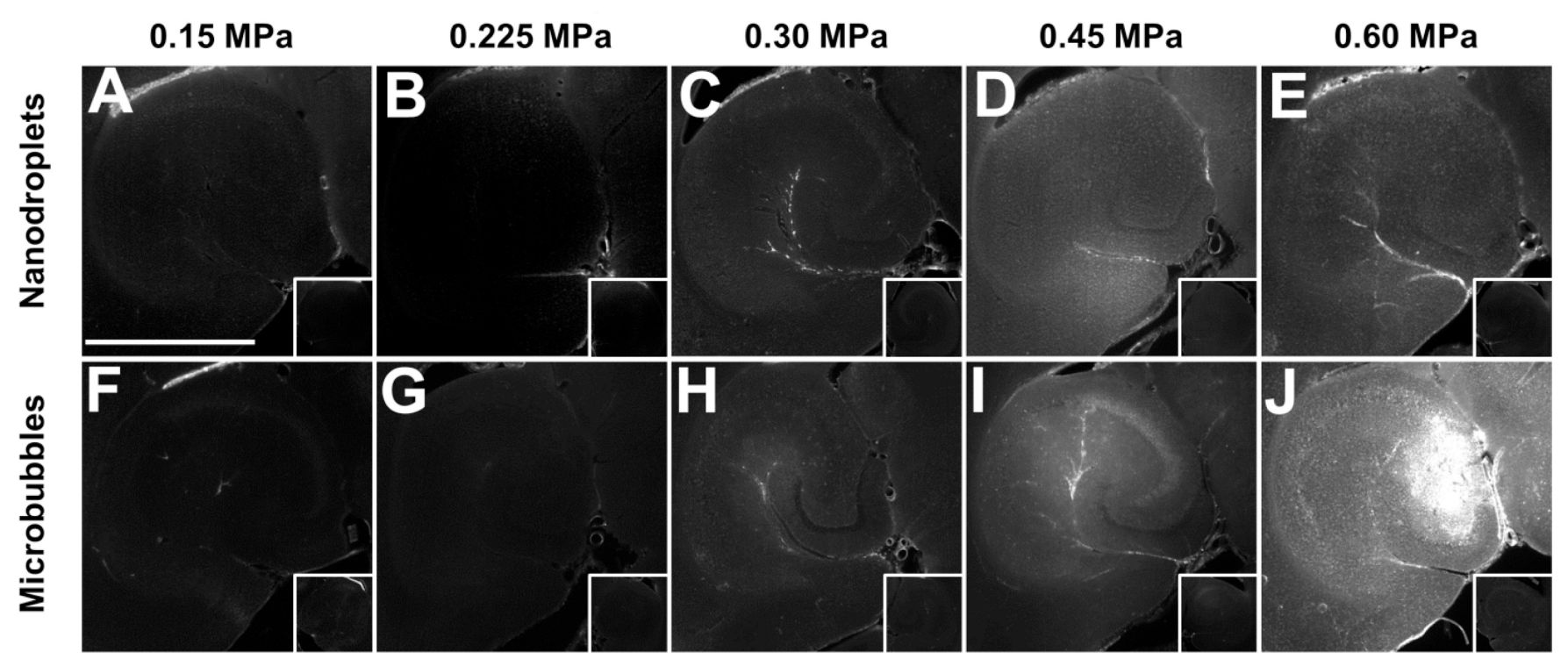

Fig. 5.

Representative fluorescence images comparing the targeted and the control (insets) hippocampi when nanodroplets (top) or microbubbles (bottom) were used to mediate BBB opening at various sonication pressures. The left hippocampus was sonicated in the presence of either nanodroplets or microbubbles and fluorescently-labeled 3-kDa dextran. Sonications were achieved at distinct peak-rarefactional pressures: $0.15 \mathrm{MPa}(\mathrm{A} \& \mathrm{~F}), 0.225 \mathrm{MPa}(\mathrm{B} \& \mathrm{G})$, $0.30 \mathrm{MPa}(\mathrm{C} \& \mathrm{H}), 0.45 \mathrm{MPa}(\mathrm{D} \& \mathrm{I})$ and $0.60 \mathrm{MPa}(\mathrm{E} \& \mathrm{~J})$. The scale bar in A depicts $1 \mathrm{~mm}$. 


\section{A}
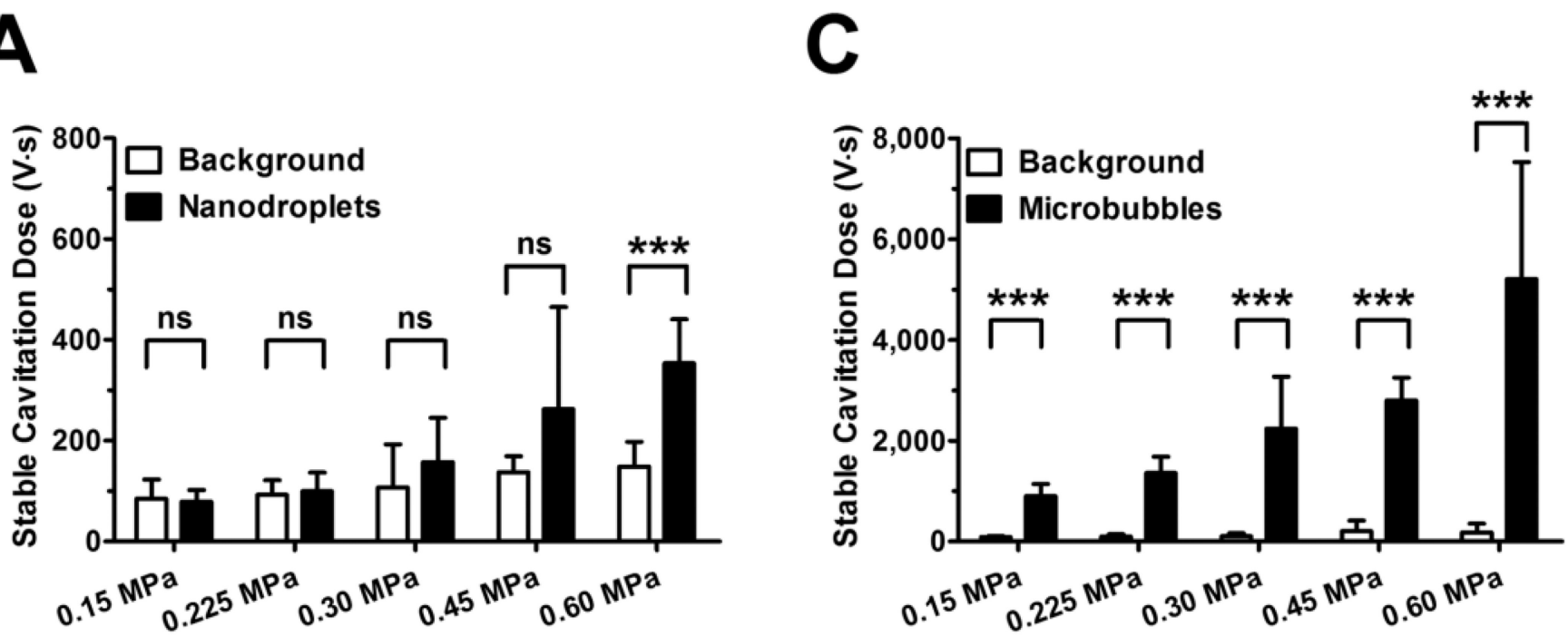

$0.15 \mathrm{MPa}_{0.225}{ }_{0.30}{ }_{0.30} \mathrm{MPa}_{0.45} \mathrm{MPa}_{0.60} \mathrm{MPa}$
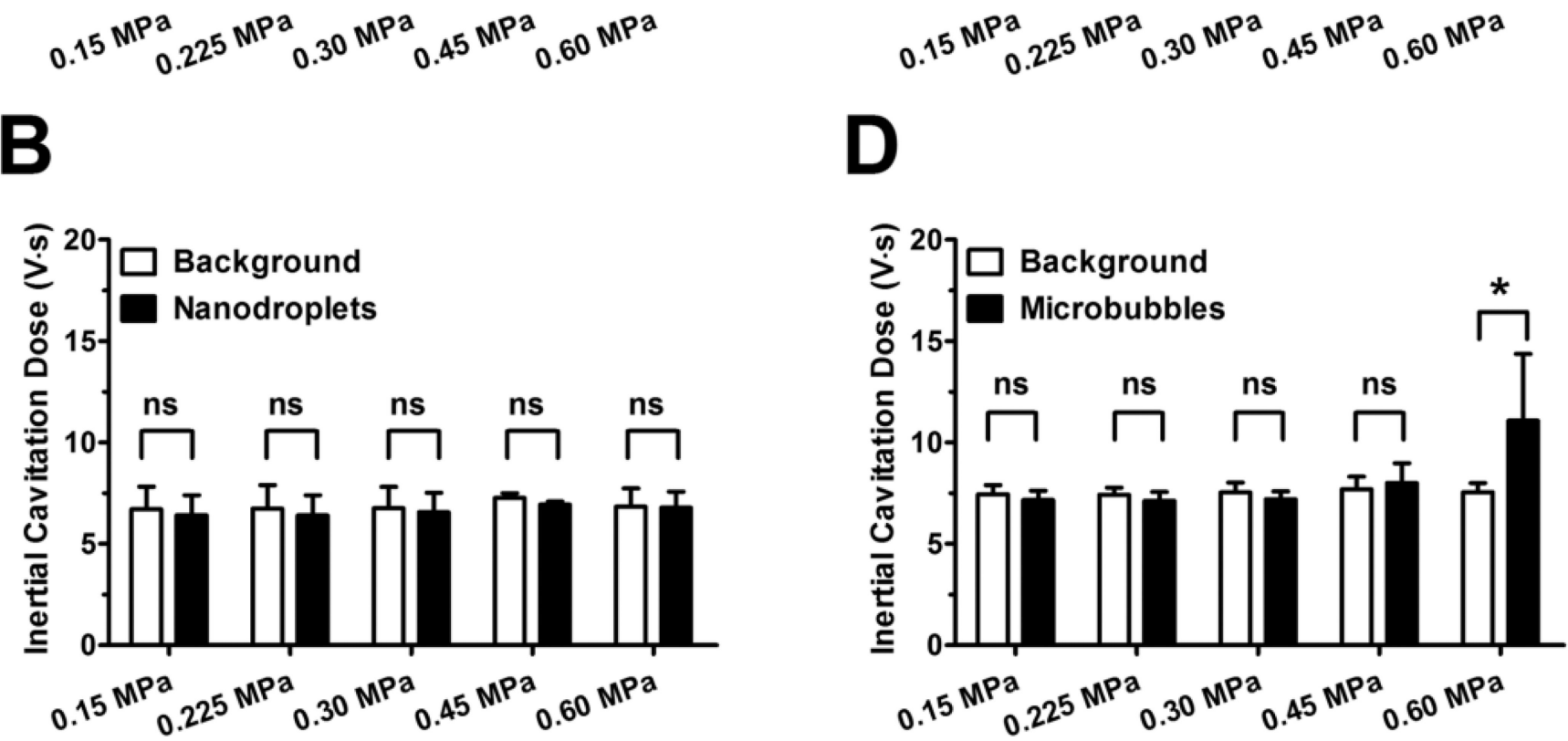

Fig. 6.

Quantified acoustic emission detected during BBB opening at various sonication pressures.

Stable cavitation implied that vaporized nanodroplets or microbubbles underwent stable nonlinear oscillation during sonication, while inertial cavitation signified particle fragmentation. Significant SCD increase was detected after nanodroplet injection at the highest pressure level (A), but no significant ICD increase was measured (B). On the other hand, significant increase of SCD was detected for all pressures after microbubble administration (C), and ICD increase was detected at the highest acoustic exposure level (D). All numbers are reported as mean \pm standard deviation. (ns: not significant; *: $\mathrm{P}<0.05$; ***: $\mathrm{P}<0.001)$ 

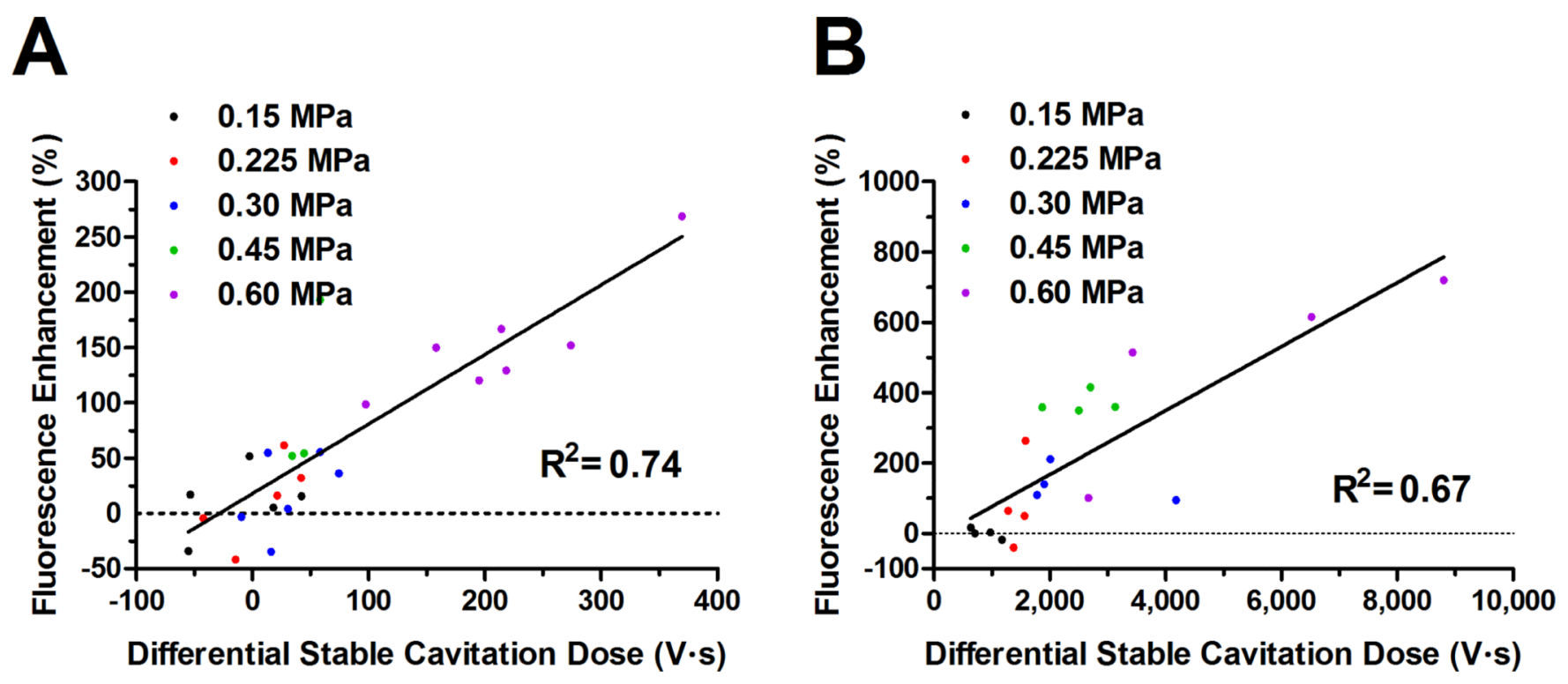

Fig. 7.

Correlation between differential stable cavitation dose (SCD) and fluorescence enhancement. The SCD was normalized based on the background emission signal measured prior to contrast agent injection. Linear correlations were obtained for both (A) nanodroplets $\left(\mathrm{R}^{2}=0.74\right)$ and $(\mathrm{B})$ microbubbles $\left(\mathrm{R}^{2}=0.67\right)$. 


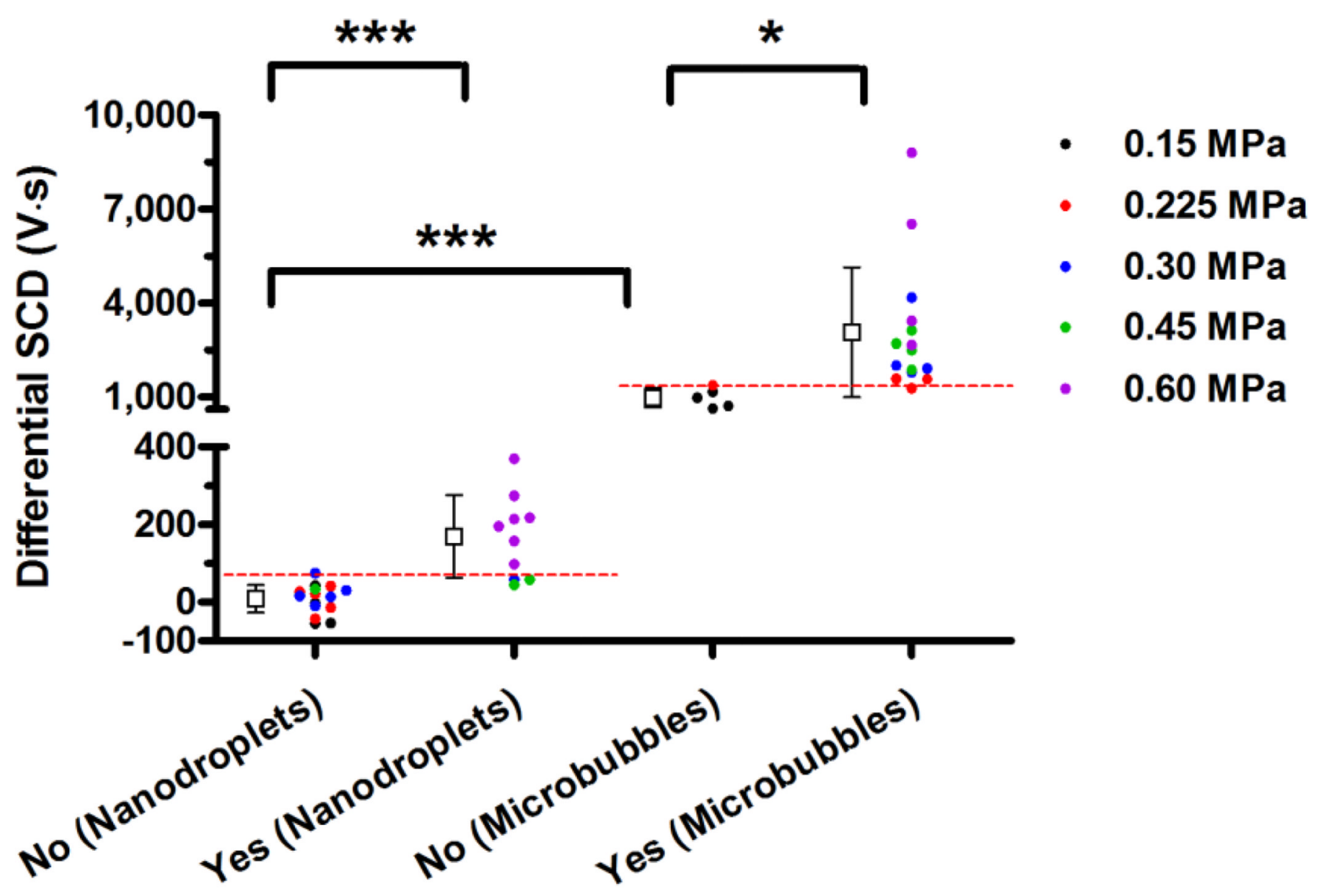

\section{BBB Opening Results}

Fig. 8.

Stable cavitation dose (SCD)-indicated BBB opening threshold. The SCD was grouped based on whether significant dextran delivery was detected. Each individual brain was concluded to have significant BBB opening when the difference in fluorescence intensity between the sonicated and the control hippocampi was two standard deviations greater than the average value obtained for the corresponding sham group. Sonication using microbubbles as the contrast agents produced a much wider range of SCD values comparing to when nanodroplets were used. The SCD threshold for predicting BBB opening appeared to be contrast agent dependent. 7 out of 10 mice showed significant dextran delivery with SCD values greater than $74 \mathrm{~V} \cdot \mathrm{s}$ using nanodroplets while $92.3 \%$ of the animals showed significant dextran delivery at SCD above $1.4 \mathrm{kV} \cdot \mathrm{s}$ using microbubbles. The slightly weaker threshold for the nanodroplet group indicated secondary mechanism other than cavitation could induce the BBB opening. $(*: \mathrm{P}<0.05 ; * * *: \mathrm{P}<0.001)$ 


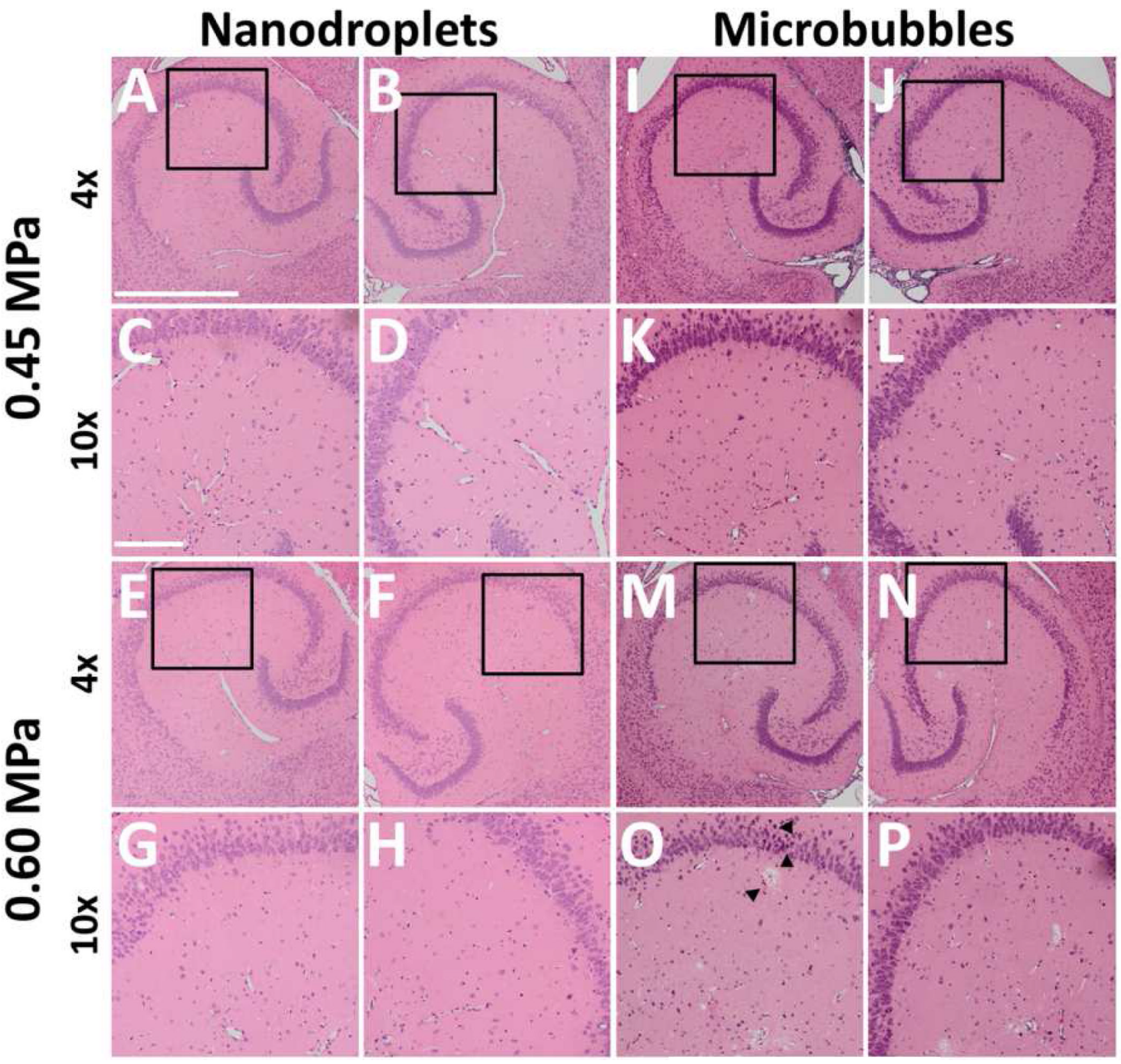

Fig. 9.

Representative histological images of the targeted and control hippocampi. The animals were sacrificed $1 \mathrm{~h}$ after sonication. (Left) No erythrocyte extravasations, dark neurons, gross hemorrhage or microvacuolations were observed when nanodroplets were used to mediate $\mathrm{BBB}$ opening at either $0.45 \mathrm{MPa}$ or $0.60 \mathrm{MPa}$. (Right) When microbubbles were used, erythrocyte extravasations or dark neurons were not seen at $0.45 \mathrm{MPa}$. However, small clusters of erythrocyte extravasations and a few dark neurons were observed (arrow heads in $\mathrm{O})$, indicating minor damage, after sonication at 0.60 MPa. The boxed regions in all $4 \times$ images are further zoomed into 10×. The scale bars in A and C depict $1 \mathrm{~mm}$ and $100 \mu \mathrm{m}$, respectively. 
Table 2

Summary of the fluorescence imaging analysis.

\begin{tabular}{cccc}
\hline $\begin{array}{c}\text { Contrast } \\
\text { agent }\end{array}$ & $\begin{array}{c}\text { Acoustic pressure } \\
\text { (MPa) }\end{array}$ & $\begin{array}{c}\text { Fluorescence } \\
\text { enhancement } \pm \text { S.D. (\%) }\end{array}$ & $\begin{array}{c}\text { Number of mice with significant dextran } \\
\text { delivery/total number of mice evaluated using } \\
\text { fluorescence microscopy }\end{array}$ \\
\hline \multirow{3}{*}{ Nanodroplet } & Sham & $21.41 \pm 25.97$ & $0 / 6$ \\
& 0.15 & $11.04 \pm 30.75$ & $0 / 5$ \\
& 0.225 & $12.73 \pm 38.81$ & $0 / 5$ \\
& 0.30 & $18.70 \pm 36.07$ & $1 / 6$ \\
& 0.45 & $91.84 \pm 67.91$ & $3 / 5$ \\
& 0.60 & $155.09 \pm 55.03$ & $7 / 7$ \\
\hline \multirow{3}{*}{ Microbubble } & Sham & $-7.67 \pm 15.41$ & $0 / 5$ \\
& 0.15 & $5.95 \pm 17.04$ & $0 / 5$ \\
& 0.225 & $72.26 \pm 114.28$ & $3 / 5$ \\
& 0.30 & $184.10 \pm 110.77$ & $5 / 5$ \\
& 0.45 & $353.64 \pm 46.23$ & $5 / 5$ \\
\hline
\end{tabular}

